\title{
两条算子遍历定理
}

设 $\mathscr{R}$ 是复的非零 Banach 空间, $A \in$ . $\mathscr{B}(\mathscr{C})$ 表示 $A$ 是 $\mathscr{T}$ 上的有界线性算子. 记 $A_{n}-\frac{1}{n} \sum_{i=0}^{n-1} A^{i}$. 如果 $\left\{A_{n}\right\}$ 一致或强 收玫, 称 $A$ 是一致或强遍历的. 如果 $\mathscr{R}=$ $\mathscr{L}^{p}$, 又如果对任何 $f \in \mathscr{L}^{p},\left\{A_{n} f\right\}$ 是几平 处处收玫的,称 $A$ 是点态遍历的. 当复数 $\lambda_{0}$ 是 $A$ 的孤立谱点, 并且是 $(\lambda I-A)^{-1}$ 的极 点时, $\lambda_{0}$ 称为 $A$ 的极点. 我们得到了下述结 果.

定理 1 设 $A \in \mathscr{B}(\mathscr{X})$. 如果对 $\mathscr{E}$ 中任一个元素 $x$,下述两个不等式成立:

$$
\sum_{n=1}^{\infty} \frac{1}{n}\left\|A_{n} x\right\|<\infty,
$$

$$
\sum_{n=1}^{\infty} \frac{1}{n}\left\|A^{n} x\right\|<\infty,
$$

则 $A$ 是强遍历的. 当 $\mathscr{X}=\mathscr{L}^{p}(1 \leqslant p \leqslant$ $\infty)$ 时, $A$ 还是点态遍历的.

定理 2 设 $A \in \mathscr{B}(\mathscr{X})$ 的谱半径不 大于 1 , 又设 $A$ 在单位圆周上的谱点都是一阶 极点. 则 $A$ 是一致遍历的. 当 $\mathscr{C}-\mathscr{L}^{\text {* }}$ $(1 \leqslant p \leqslant \infty)$ 时, $A$ 还是点态遍历的.

注记凡论及 $\mathscr{L}^{p}$ 之处, 均假定相应 的测度空间是 $\sigma$-有限的.

陶志光

(广西大学数学系,南宁 530004)

\section{关于 Hayman 的一个问题*}

本文采用值分布理论的标准记号, $B$ 为 论度有限的正实数例外集.

1976 年, Frank 在文献 [1] 中证明了 Hayman 的一个问题 ${ }^{[2]}$ : 对于亚纯函数 $f$ 及 $k \geq 2$, 如果 $f$ 及 $f^{(k)}$ 没有零点, 那么 $f(z)=$ $\exp (a x+b)$ 或 $f(x)=(a z+b)^{-n}$, 其中 $a \neq 0$ 及 $b$ 是常数, $n$ 是正整数.

这个问题可以变得更为一般,如果 $f^{(k)}$ 用 线性微分算子

$$
L(f)=f^{(k)}+a_{k-2} f^{(k-2)}+\cdots+a_{0} f
$$

所代替. 当 $L(f)$ 的系数是多项式及 $f$ 是整 函数时,这个新问题已被 Frank 和 Hellers$\operatorname{tein}^{(3)}$ 完全解决. 当 $f$ 是亚纯函数时, 他们 在文献 [3] 中证明了

定理 $\mathbf{A}$ 设 $k \geqslant 3, f$ 是平面上的
亚纯函数, $L(f)$ 由(1)式给出, 式中的系数 $a_{j}(j=0, \cdots, k-2)$ 是多项式. 如果 $f$ 及 $L(f)$ 仅有有限个零点, 那么

$$
T\left(r, f^{\prime} / f\right)=O\left(r^{\lambda}\right), r \in E,
$$

其中 $\lambda=1+\max _{0 \leqslant j<k-2} \frac{\operatorname{deg} a_{j}}{k-j}$. 如果所有 $a_{i}$ 恒等于零, 则(2)式中的 $r^{2}$ 可用 $\log r$ 代替. 另外,当 (1)式中的系数 $a_{i}$ 都是常数时, Steinmetz ${ }^{2}$ 证明了

定理 $\mathrm{B}$ 设 $k \geqslant 3, f$ 是平面上的亚 纯函数, $L(f)$ 由(1)式给出, 式中 $a_{j}(j=$ $0, \cdots, k-2)$ 都是常数. 如果 $f \cdot L(f) \neq 0$,

* 国家自然科学基金资助项目.

1) Steinmetz, N., On the zeros of $\left(f^{(D)}+a_{p-1}\right.$ $\left.f^{(p-1)}+\cdots+a_{0} f\right) f$, to appear.

通报

1991 年 